\title{
Onagraceae endémicas del Perú
}

\section{Susy Castillo ${ }^{1}$, Christhian Monsalve ${ }^{1}$ y Blanca León ${ }^{1,2}$}

\begin{abstract}
${ }^{1}$ Museo de Historia Natural, Av. Arenales 1256, Aptdo. 14-0434, Lima 14, Perú.

susy_827@yahoo.com

christhianml@hotmail.com

2 Plant Resources Center, University of Texas at Austin, Austin TX 78712 EE.UU.

blanca.leon@mail.utexas.edu
\end{abstract}

\section{Resumen}

Esta es la familia de las «fucsias», la cual es reconocida en el Perú por presentar seis géneros y 76 especies (Brako \& Zarucchi, 1993; Ulloa Ulloa et al., 2004), principalmente arbustos y hierbas. En este trabajo reconocemos 24 especies endémicas, la mayoría en el género Fuchsia. Las especies endémicas se encuentran mayormente en la región Bosques Muy Húmedos Montanos, entre los 1600 y 2500 m de altitud. Diez especies endémicas se encuentran representados dentro del Sistema Nacional de Áreas Naturales Protegidas por el Estado.

Palabras claves: Onagraceae, Fuchsia, Perú, endemismo, plantas endémicas.

\section{Abstract}

This is the "fuchsia» family, which is represented in Peru by six genera and 76 species (Brako \& Zarucchi, 1993; Ulloa Ulloa et al., 2004), mostly shrubs and herbs. Here we recognize 24 endemic species, most of them in the genus Fuchsia. These endemic species are found mainly in the Very Humid Montane Forests region, between 1600 and $2500 \mathrm{~m}$ elevation. Ten endemic species have been recorded in the Peruvian System of Protected Natural Areas.

Keywords: Onagraceae, Fuchsia, Peru, endemism, endemic plants.

\section{Fuchsia abrupta I.M. Johnst.}

\section{LC}

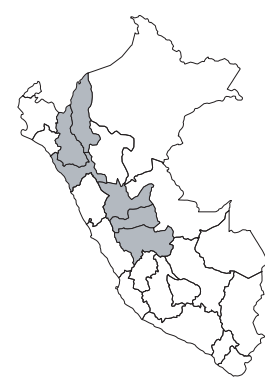

Publicación: Contr. Gray Herb. 75: 37. 1925.

Colección tipo: J.F. Macbride 4541

Herbarios: F, GH, K, US.

Nombre común: Desconocido.

Registro departamental: AM, CA, HU, JU, LL, PA.

Regiones Ecológicas: BMHM; 1650$2860 \mathrm{~m}$.

SINANPE: PNO, PNYC

Herbarios peruanos: HUT (6), USM (17).

Observaciones: Arbusto trepador conocido de varias localidades en el norte y centro del país, en la vertiente oriental. Ha sido recolectada en varias subcuencas del Huallaga y del Perené.

\section{Fuchsia austromontana I.M. Johnst.}

LC

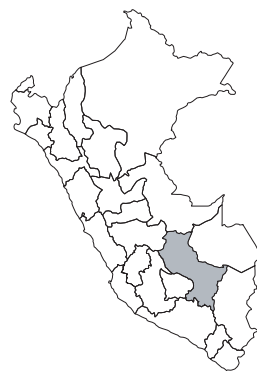

Publicación: J. Arnold Arbor. 20: 242. 1939.

Colección tipo: J. West 7083

Herbarios: GH, UC.

Nombre común: Desconocido.

Registro departamental: CU.

Regiones Ecológicas: BPM, BMHM; 2200-3800 m.

SINANPE: PNM

Herbarios peruanos: USM (8).

Observaciones: Arbusto epífito conocido de poblaciones, principalmente en ambientes montanos, en el Parque Nacional Manu y alrededores. Sus poblaciones han sido registradas consecutivamente en cada década desde los 1930. Por su hábito requiere de ambientes con cobertura boscosa y su área de presencia se extienda, tal vez, en Cusco y Puno. Será necesario evaluar en el futuro su presencia en otras localidades y las amenazas a su hábitat.

\section{Fuchsia ceracea P.E. Berry}

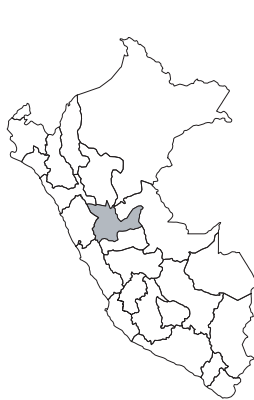

CR, B1ab(iii)

Publicación: Ann. Missouri Bot. Gard. 69(1): 149-150. f. 31, 62. 1982.

Colección tipo: P.E. Berry \& J. Aronson 3081

Herbarios: $\mathrm{MO}$

Nombre común: Desconocido.

Registro departamental: HU.

Regiones Ecológicas: BMHM; $2500 \mathrm{~m}$.

SINANPE: Sin registro.

Herbarios peruanos: Ninguno.

Observaciones: Arbusto conocido aparentemente sólo de la colección tipo, una planta recolectada en 1978, en los alrededores de Carpish. Esta localidad alberga numerosos endemismos, sin embargo, no recibe protección oficial. Amenazas a esta especie podrían estar asociadas a la deforestación que reduce no sólo hábitat, sino también modifica las condiciones ambientales de los bosques.

\section{Fuchsia cestroides Schulze-Menz}

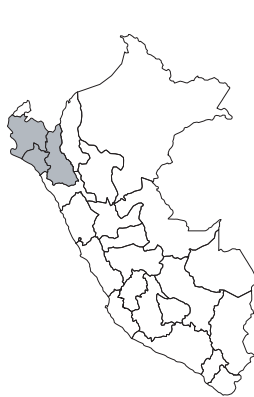

EN, B1ab(iii)

Publicación: Notizbl. Bot. Gart. BerlinDahlem 15: 137. 1940.

Colección tipo: A. Weberbauer 6423

Herbarios: B, F, GH, US.

Nombre común: Desconocido.

Registro departamental: CA, LA, PI.

Regiones Ecológicas: BS; $1100-2200 \mathrm{~m}$. SINANPE: Sin registro.

Herbarios peruanos: HAO (1).

Observaciones: Arbusto caducifolio, conocido solamente del noroccidente del país. El ejemplar tipo fue recolectado en 1912 y vuelto a reubicar en 1991. Habita bosques secos, los que hasta hace poco han sido escasamente herborizados. 


\section{Fuchsia chloroloba I.M. Johnst.}

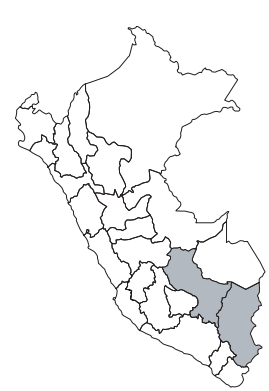

\section{NT}

Publicación: J. Arnold Arbor. 20: 243. 1939. Colección tipo: F.W. Pennell 13973

Herbarios: F, GH, NY, PH.

Nombre común: Desconocido.

Registro departamental: CU, PU.

Regiones Ecológicas: BMHM; 1600$3350 \mathrm{~m}$.

SINANPE: PNM

Herbarios peruanos: USM (4).

Observaciones: Arbusto epífito, ramoso con tubérculos, conocido de varios ejemplares, provenientes mayormente de los alrededores y del área del Parque Nacional Manu. Otras poblaciones conocidas se hallan en las cuencas del Apurímac y del Inambari.

\section{Fuchsia confertifolia Fielding \& Gardner}

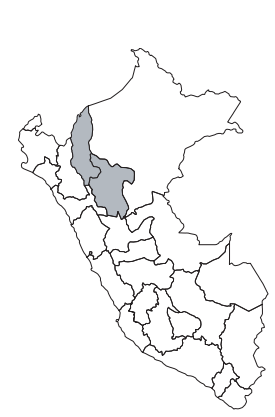

\section{EN, B1a}

Publicación: Sert. Pl. 1: pl. 28. 1844.

Colección tipo: A. Mathews 1478

Herbarios: K, OXF.

Nombre común: Desconocido.

Registro departamental: AM, SM.

Regiones Ecológicas: BMHM; 2600$3200 \mathrm{~m}$.

SINANPE: Sin registro.

Herbarios peruanos: USM (1).

Observaciones: Arbusto conocido del norte del país, de las cuencas del Mayo y del Utcubamba. La colección tipo fue recolectada en el siglo XIX, de una localidad hoy reconocida en San Martín. Podría estar representada en el Bosque de Protección del Alto Mayo.

\section{Fuchsia coriacifolia P.E. Berry}

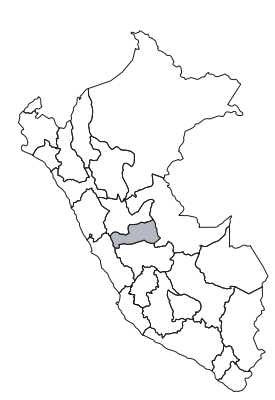

\section{CR, B1a}

Publicación: Ann. Missouri Bot. Gard. 69(1): 150-152, f. 62. 1982.

Colección tipo: R.W. Pearce 565

Herbarios: K.

Nombre común: Desconocido.

Registro departamental: PA.

Regiones Ecológicas: BMHM; altitud desconocida.

SINANPE: Sin registro.

Herbarios peruanos: Ninguno.

Observaciones: Arbusto conocido solamente de la colección tipo, una planta recolectada en el siglo XIX, de la cuenca del Pozuzo. Aparentemente no existen recolectas adicionales.

\section{Fuchsia ferreyrae P.E. Berry}

\section{VU, B1ab(iii)}

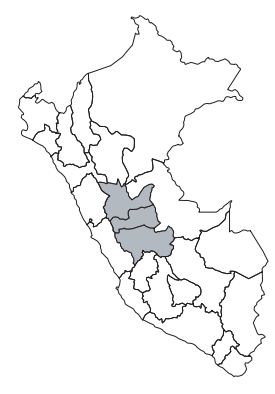

Publicación: Ann. Missouri Bot. Gard. 69(1): 79—80, f. 27, 55. 1982.

Colección tipo: P.E. Berry \& J. Aronson 3073

Herbarios: MO, US; USM.

Nombre común: Desconocido.

Registro departamental: HU, JU, PA.

Regiones Ecológicas: BMHM; 2750$3110 \mathrm{~m}$.

SINANPE: PNYC

Herbarios peruanos: AMAZ (1), USM (isotipo).
Observaciones: Arbusto trepador, conocido del centro del país, de las cuencas del Pachitea y Perené. El tipo de esta especie fue recolectado en 1978, en los bosques montanos orientales de Satipo, pero aparentemente no ha vuelto a ser recolectada ahí.

\section{Fuchsia fontinalis J.F. Macbr.}

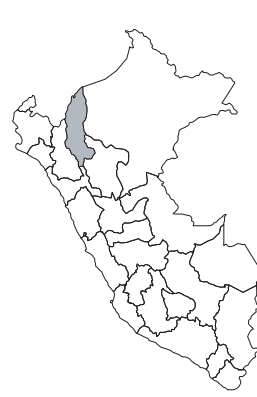

$$
\text { VU, B1a }
$$

Publicación: Candollea 8: 25. 1940.

Colección tipo: A. Mathews s.n.

Herbarios: G-DEL.

Nombre común: Desconocido.

Registro departamental: AM.

Regiones Ecológicas: BMHM; 2550$3400 \mathrm{~m}$.

SINANPE: Sin registro.

Herbarios peruanos: HAO (2), USM (7).

Observaciones: Subarbusto trepador, conocido de varias localidades, en la cuenca del Utcubamba. El tipo de esta especie fue recolectado en el siglo XIX. Habita bosques o matorrales montanos. Está presente en los Cerros Calla Calla, una localidad que alberga varios otros endemismos.

\section{Fuchsia huanucoensis P.E. Berry}

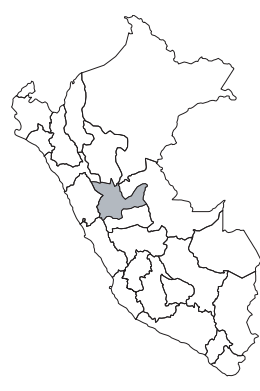

$$
\text { CR, B1ab(iii) }
$$

Publicación: Ann. Missouri Bot. Gard. 72(2): 235. 1985

Colección tipo: C. Sandeman 5166

Herbarios: K, OXF.

Nombre común: Desconocido.

Registro departamental: HU.

Regiones Ecológicas: BMHM; $2550 \mathrm{~m}$.

SINANPE: Sin registro.

Herbarios peruanos: Ninguno.

Observaciones: Arbusto conocido, aparentemente, sólo de la colección tipo, una planta recolectada en 1945, de la cuenca del Chinchao, un afluente del Huallaga. La localidad original, Carpish, incluye varios otros endemismos y debería recibir mayor atención a su conservación. Amenazas a esta especie están vinculadas a deforestación.

\section{Fuchsia inflata Schulze-Menz}

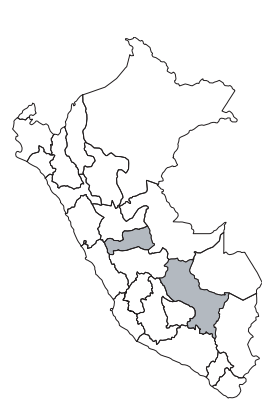

\section{VU, B1a}

Publicación: Notizbl. Bot. Gart. BerlinDahlem 15: 136. 1940.

Colección tipo: A. Weberbauer 6935

Herbarios: F, US.

Nombre común: Desconocido.

Registro departamental: CU, PA.

Regiones Ecológicas: BMHM; 2380$3300 \mathrm{~m}$.

SINANPE: PNM, SHMP, PNYC

Herbarios peruanos: HUT (1), USM (2).

Observaciones: Arbusto trepador y caducifolio, conocido del suroriente del país y de una población disyunta en Pasco. Por ello, probablemente su extensión geográfica sea mayor en los bosques montanos. El ejemplar tipo fue recolectado en 1914, de una localidad en el Parque Nacional Manu, y en donde ha vuelto a ser recolectada en diferentes años. 


\section{Fuchsia llewelynii J.F. Macbr.}

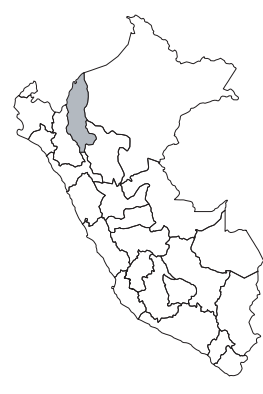
$3300 \mathrm{~m}$.

\section{EN, B1ab(iii)}

Publicación: Field Mus. Nat. Hist., Bot. Ser. 13(4): 556. 1941.

Colección tipo: L. Williams 7594

Herbarios: F, NY, UC.

Nombre común: Desconocido.

Registro departamental: AM.

Regiones Ecológicas: BMHM; 2600-

SINANPE: ZRCC

Herbarios peruanos: MOL (1).

Observaciones: Arbusto conocido de la cuenca del Utcubamba. Esta especie es conocida de la Zona Reservada Cordillera de Colán, una zona que alberga varios endemismos. Aparentemente no ha vuelto a ser recolectada desde 1978.

\section{Fuchsia macropetala C. Presl}

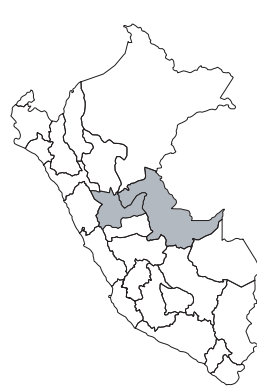

\section{EN, B1a}

Publicación: Reliq. Haenk. 2(1): 28. 1831. Colección tipo: T. Haenke s.n. Herbarios: PR.

Nombre común: Desconocido.

Registro departamental: HU, UC.

Regiones Ecológicas: BMHM; 1620$1760 \mathrm{~m}$.

SINANPE: Sin registro.

Herbarios peruanos: USM (1).

Observaciones: Arbusto conocido del centro-oriente del país, en la zona montana entre el Huallaga y el Aguaytía. Podría estar representada en el Parque Nacional Cordillera Azul. Habita ambientes intervenidos en bordes de bosque.

\section{Fuchsia mathewsii J.F. Macbr.}

\section{LC}

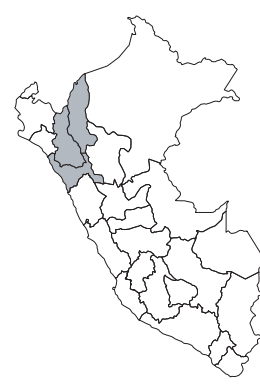

Publicación: Candollea 8: 24. 1940. Colección tipo: A. Mathews s.n. Herbarios: G-BOIS, G-DEL. Nombre común: Desconocido. Registro departamental: AM, CA, LL. Regiones Ecológicas: BPM, BMHM; 2930-3700 m.

SINANPE: Sin registro.

Herbarios peruanos: CPUN (3), HAO (2), MOL (1), USM (6).

Observaciones: Arbusto conocido solamente del norte del país, de las cuencas del Marañón y Utcubamba. Berry (1982) comentó que las poblaciones son localmente frecuentes a dispersas. Habita fragmentos de bosque montano; matorrales ribereños y otros ambientes modificados.

\section{Fuchsia ovalis Ruiz \& Pav.}

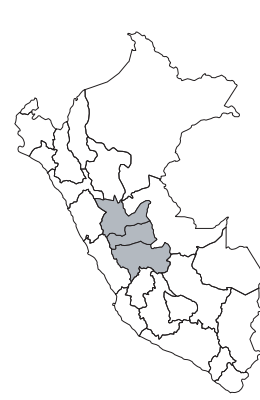

VU, B1a

Publicación: Fl. Peruv. 3: 87, pl. 324, f. a. 1802.

Colección tipo: H. Ruiz \& J. Pavón s.n.

Herbarios: MA.

Nombre común: Desconocido.

Registro departamental: HU, JU, PA.

Regiones Ecológicas: BPM, BMHM, BMHP; 750-3200 m.

SINANPE: Sin registro.

Herbarios peruanos: USM (10)
Observaciones: Subarbusto conocido del centro del país. Fue descrita de una planta recolectada en el siglo XVIII, de la cuenca del Huallaga, en donde ha vuelto a ser registrada. Poblaciones adicionales se conocen también de las cuencas del Perené y Pachitea.

\section{Fuchsia pachyrrhiza P.E. Berry \& B.A. Stein}

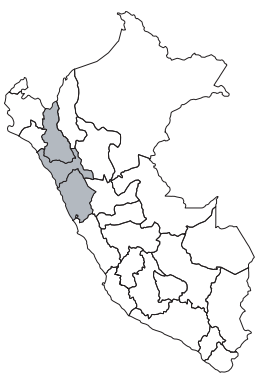
EN, B1ab(iii)

Publicación: Syst. Bot. 13(4): 483—492. 1988. Colección tipo: B. Stein et al. 4066 Herbarios: MO, US, WISC.

Nombre común: Desconocido. Registro departamental: AN, CA, LL. Regiones Ecológicas: MA; $1870-2800 \mathrm{~m}$. SINANPE: Sin registro.

Herbarios peruanos: HAO (2), USM (3).

Observaciones: Planta leñosa conocida solamente de la vertiente del Pacífico, de las cuencas del Chicama y Magdalena. Esta especie ocupa matorrales en quebradas húmedas y roquedales protegidos.

\section{Fuchsia pilosa Fielding \& Gardner}

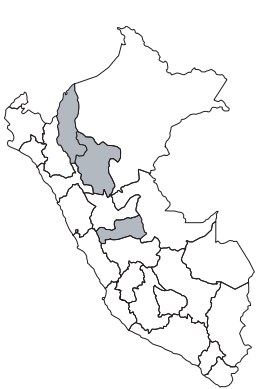

\section{EN, B1a}

Publicación: Sert. Pl. 1: pl. 27. 1844.

Colección tipo: A. Mathews s.n.

Herbarios: BM, CGE, K, OXF.

Nombre común: Desconocido.

Registro departamental: AM, PA, SM.

Regiones Ecológicas: BMHM; 2300$2400 \mathrm{~m}$.

SINANPE: Sin registro.

Herbarios peruanos: USM (2).

Observaciones: Subarbusto conocido de unas cinco poblaciones, en las cuencas del Marañón, Mayo y Pachitea. El ejemplar tipo fue recolectado en el siglo XIX y probablemente provenía de la cuenca del Marañón. Esta especie ha sido recolectada en bordes de bosque montano intervenido.

\section{Fuchsia rivularis J.F. Macbr.}

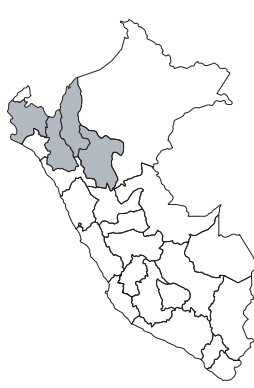

\section{VU, B1ab(iii)}

Publicación: Candollea 8: 24. 1940.

Colección tipo: A. Mathews s.n.

Herbarios: G.

Nombre común: Desconocido.

Registro departamental: AM, CA, SM. Regiones Ecológicas: BMHM; 1700$2750 \mathrm{~m}$.

SINANPE: PNC

Herbarios peruanos: CPUN (1), HAO (1), USM (8).

Observaciones: Arbusto conocido de por lo menos seis poblaciones, en el norte del país, de las cuencas del Marañón y Utcubamba. Habita laderas rocosas y fragmentos de bosque montano. Probablemente amenazas a sus poblaciones podrían estar asociadas a deforestación.

\section{Fuchsia sanmartina P.E. Berry}

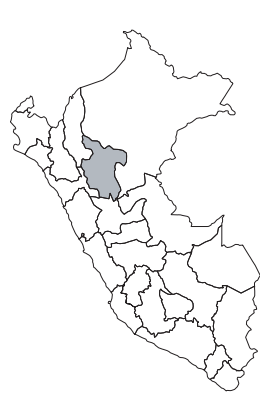
EN, B1ab(iii)

Publicación: Ann. Missouri Bot. Gard. 69(1): 152-153, f. 62. 1982.

Colección tipo: A.C. Hamilton \& P.M. Holligan Herbarios: K, MO, NY, S, UC.

Nombre común: Desconocido.

Registro departamental: SM.

Regiones Ecológicas: BPM, BMHM; $3100-3450 \mathrm{~m}$.

SINANPE: PNRA

Herbarios peruanos: HUT (2), USM (4). 
Observaciones: Arbusto conocido solamente del nor-oriente del país, de localidades ubicadas dentro del Parque Nacional Río Abiseo. Ha sido recolectada en dos de los valles que conforman este parque, estas poblaciones ocupan ambientes ecotonales bosque-pajonal.

\section{Fuchsia simplicicaulis Ruiz \& Pav.}

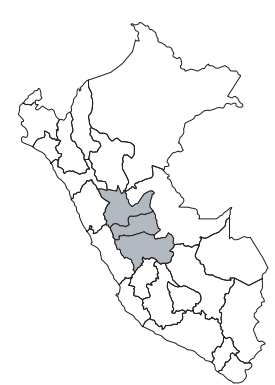

\section{VU, B1a}

Publicación: Fl. Peruv. 3: 89, pl. 322, f. a 1802.

Colección tipo: H. Ruiz \& J. Pavón 11/92

Herbarios: MA.

Nombre común: Desconocido.

Registro departamental: HU, JU, PA.

Regiones Ecológicas: BMHM; 1750$2500 \mathrm{~m}$.

SINANPE: Sin registro.

Herbarios peruanos: USM (9).

Observaciones: Arbusto trepador conocido de varias localidades en el centro del país, en donde ocupa matorrales y márgenes de bosque intervenidos. Incendios intencionales podrían afectar a sus poblaciones.

\section{Fuchsia tincta I.M. Johnst.}

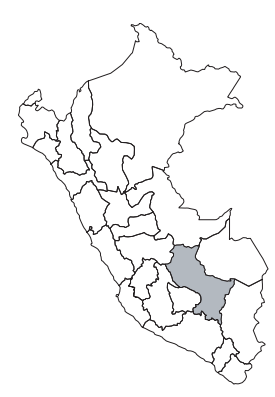

\section{EN, B1ab(iii)}

Publicación: J. Arnold Arbor. 20: 242. 1939.

Colección tipo: J. West 7092

Herbarios: GH, MO, UC.

Nombre común: Desconocido.

Registro departamental: CU.

Regiones Ecológicas: BPM, BMHM; 1800-2850 m.

SINANPE: PNM, SHMP

Herbarios peruanos: USM (8).

Observaciones: Arbusto trepador conocido de unas pocas localidades, la mayoría de ellas ubicadas en la cuenca alta del Alto Madre de Dios. Esta especie ocupa bosques montanos fragmentados. Una población adicional es conocida de la cuenca del Urubamba. Especie simpátrica con Fuchsia vargasiana.

\section{Fuchsia vargasiana Munz ex Vargas}

EN, B1ab(iii)

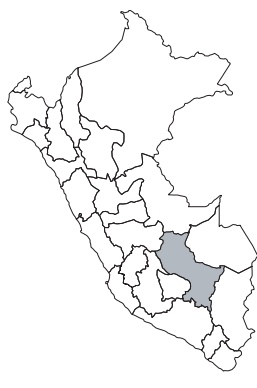

Publicación: Diez Anos Serv. Bot. Univ. Cuzco 50, f. 16. 1946.

Colección tipo: C. Vargas C. 73

Herbarios: BH, F, MO, POM, UC; CUZ.

Nombre común: Desconocido.

Registro departamental: CU.

Regiones Ecológicas: BMHM; 1900$2500 \mathrm{~m}$.

SINANPE: PNM, SHMP

Herbarios peruanos: CUZ (isotipo citado), USM (8).

Observaciones: Arbusto escandente, conocido de las cuencas del Alto Madre de Dios y del Urubamba. Esta es una especie simpátrica con Fuchsia tincta, también una endémica.

\section{Fuchsia wurdackii Munz}

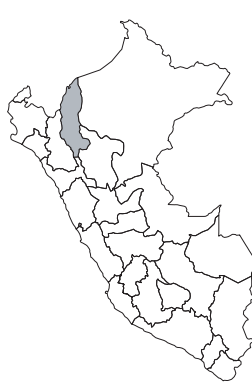

EN, B1ab(iii)

Publicación: Brittonia 16: 229. 1964.

Colección tipo: J.J. Wurdack 622

Herbarios: US; USM!

Nombre común: Desconocido.

Registro departamental: AM.

Regiones Ecológicas: BMHM; 2200$2400 \mathrm{~m}$.

SINANPE: Sin registro.

Herbarios peruanos: USM (isotipo+2).

Observaciones: Arbusto trepador, conocido solamente del norte del país, en un área entre las cuencas del Utcubamba y Marañón. Deforestación e incendios intencionales podrían ser amenazas para sus poblaciones.

\section{Oenothera featherstonei Munz \& I.M. Johnst.}

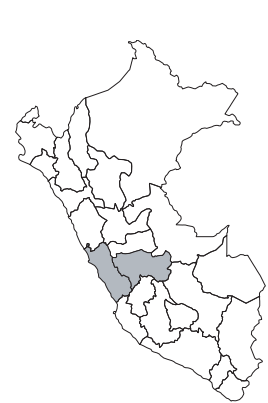

\section{VU, B1a}

Publicación: Contr. Gray Herb. 75: 19. 1925.

Colección tipo: J.F. Macbride \& W. Featherstone 270

Herbarios: F.

Nombre común: Desconocido.

Registro departamental: JU, LI.

Regiones Ecológicas: DST, MDE, MA; $400-2500 \mathrm{~m}$.

SINANPE: Sin registro.

Herbarios peruanos: USM (7).

Observaciones: Hierba postrada, conocida del centro del país, de varias localidades. El ejemplar tipo proviene de la cuenca del Rímac, en donde fue recolectado en 1922. Otras poblaciones son conocidas también de la cuenca del Chillón. 